C. Fernandes, A. J. Pontes, J. C. Viana, A. Gaspar-Cunha*

Institute for Polymer and Composites, Dept. of Polymer Engineering, University of Minho, Portugal

\title{
Using Multi-objective Evolutionary Algorithms for Optimization of the Cooling System in Polymer Injection Molding
}

The cooling process in polymer injection molding is of great importance as it has a direct impact on both productivity and product quality. In this paper a Multi-objective Optimization Genetic Algorithm, denoted as Reduced Pareto Set Genetic Algorithm with Elitism (RPSGAe), was applied to optimize both the position and the layout of the cooling channels in the injection molding process. The optimization model proposed in this paper is an integration of genetic algorithms and Computer-Aided Engineering, CAE, technology applied to polymer process simulations. The main goal is to implement an automatic optimization scheme capable of defining the best position and layout of the cooling channels and/or setting the processing conditions of injection moldings. In this work the methodology is applied to an L-shape molding with the aim of minimizing the part warpage quantified by two different conflicting measures. The results produced have physical meaning and correspond to a successful process optimization.

\section{Introduction}

The injection molding cycle consists of three important stages: mold filling, melt packing, and part cooling. The cooling system design is of considerable importance since about 60 to $70 \%$ of the cycle time is taken up by the cooling phase. An efficient cooling system design aiming at reducing cycle time must minimize undesired defects such as sink marks, part warpage and differential shrinkage.

In cooling system design, the design variables typically include the size of cooling channel and its layout, the thermal properties, temperature and flow rate of the coolant fluid. With so many design parameters involved, the determination of the optimum cooling system is extremely difficult. For an optimum design, the designer needs a powerful tool integrating the cooling analysis and optimization programs into the design process. Therefore, it is necessary to develop computer-based methods to achieve efficient cooling system designs that opti-

\footnotetext{
* Mail address: António Gaspar-Cunha, IPC/I3N- Institute for Polymer and Composites, Dept. of Polymer Engineering, University of Minho, Campus de Azurém, 4800-058 Guimarães, Portugal

E-mail: agc@dep.uminho.pt
}

mize channel dimensions and layout as well as the processing conditions. The application of computer tools on the various designing stages of the injection molding process is very frequent (e.g., Duff, 2000; Menges and Mohren, 1986). Usually, the sequential steps of this design process are the following: definition of a finite element mesh representative of the part geometry and cooling system (in the case of a cooling analysis), selection of materials, definition of the gate location and of the initial processing variables. Finally, after launching the simulation the outputs are analysed. Various optimization strategies using different methodologies to optimize the shape and locations of cooling channels in injection molding have been reported in the literature (Tang et al., 1997, 1998; Park and Kwon, 1998a, 1998b, 1998c; Mathey et al., 2004; Lam et al., 2004; Qiao, 2006; Pirc et al., 2008a and Pirc et al., 2008b). Tang et al. (1997 and 1998) used 2D transient Finite Element Method (FEM) simulations coupled with Powell's optimization method to optimize the cooling channel geometry to get uniform temperature in the polymer part. Park and Kwon (1998a) developed 2D and 3D stationary Boundary Element Method (BEM) simulations in the injection molds coupled with $1 \mathrm{D}$ transient analytical computation in the polymer part (throughout the molding thickness). The heat transfer integral equation is differentiated to get sensitivities of a cost function to the parameters (Park and Kwon, 1998b). The calculated sensitivities are then used to optimize the position of linear cooling channels for simple layouts (Park and Kwon, 1998c). Mathey et al. (2004) developed an optimization procedure to improve cooling of injection molds. The model uses a mathematical programming method, sequential quadratic programming (SQP), to modify and improve automatically the geometry and the process parameters according to an objective function (e. g. cooling time or temperature uniformity). The SQP method was coupled with the BEM to solve thermal problems of cooling during injection. Lam et al. (2004) explored an approach to optimize both cooling channel design and process condition selection simultaneously through an evolutionary algorithm. The design variables used were: co-ordinates of centres of cooling channels, sizes of cooling channels, flow rate and inlet temperature of the coolant in each cooling channel, packing time, cooling time and mold opening time. The work integrated genetic algorithm and CAE tool with the objective of achieving the most uniform cavity surface tempera- 
ture to assure product quality. Qiao (2006) implemented a systematic computer-aided methodology for the optimization of cooling system design. Cycle-averaged cooling analysis, perturbation-based sensitivity analysis, and the hybrid simulated annealing and Davidon-Fletcher-Powel method optimizer were applied to search for the optimal design. Significant uniformity of the temperature distribution along the cavity surface was obtained as a result of the optimization process. Pirc et al. (2008a) used BEM and Dual Reciprocity Method (DRM) applied to unsteady heat transfer of injection molds. The BEM code was combined with an adaptive reduced modelling, and applied to a practical methodology for optimizing both the position and the layout of the cooling channels in injection molding process. For that, the direct computation was coupled with an optimization algorithm such as SQP, where a potential problem is defined in a $2 \mathrm{D}$ unbounded domain. Later, Pirc et al. (2008b) extended that methodology to optimize both the position and the layout of the cooling channels in 3D simulations of the injection molding process. The optimization variables used were the position and shape parameters of the cooling channels and the outputs were the temperature of the molded part surface and the variation of the temperature along this surface.

An important limitation of these optimization methodologies resides in the use of single objective optimization strategies, where the various objectives are either optimized alone or using an aggregation function. This type of methodology is not able to capture the trade-off between the objectives, which can bias the solution found. Therefore, an automatic optimization methodology based on Multi-Objective Evolutionary Algorithm - MOEA (Gaspar-Cunha and Covas, 2004) to define the best position and layout of cooling channels and/or defining the values of important operating conditions in injection molding is proposed in this work. For that purpose a MOEA is linked to an injection molding simulator code (in this case C-MOLD). The methodology proposed here is general enough to be used with any injection molding simulator code. The limitation of their applicability lies on the necessity of communicating the data in both directions (i.e., from the MOEA to the simulator and from the simulator to the MOEA). The proposed optimization methodology was applied to a case study where the layout of the cooling channels and/or the processing conditions are established in order to minimize the part warpage quantified by two conflicting objectives as described below. This methodology proposed here is general enough to be used with any other injection molding simulator code.

\section{Development of the Optimization System}

\subsection{Framework}

In this work a methodology integrating computer simulations of the injection molding process, an optimization methodology based on Evolutionary Algorithm (EA) and multi-objective criteria is proposed. This methodology is used to establish the configurations of the cooling circuits and/or define the best processing conditions that lead to a part with lower warpage. EAs are a class of metaheuristics based on the concepts of the natural evolutions. The selection, crossover and mutation op- erators are applied to the current population that evolves during the successive generations (or iterations). The initial generation of chromosomes (initial population) indicating the configurations of the cooling circuits and/or the set of operative processing variables is randomly generated within the feasible search space and evaluated by the C-MOLD modelling routine. The quality of the cooled part is quantified by the fitness function (angle deformation) of each chromosome. Then a new generation is produced though EA reproduction and re-evaluated. The process iterates until an optimal or near optimal cooling system design and/or processing conditions are found.

Figure 1 shows the interface for integrating C-MOLD and the EA-based optimization routine. A design with cooling circuit coordinates and/or processing conditions are sent to $\mathrm{C}-\mathrm{MOLD}$ and shrinkage and warpage analysis is done through command files provided by C-MOLD software. When the analysis is finished, the optimization routine will read the C-MOLD results and calculations are done to measure the deformation angle along the part.

\subsection{MOEA}

Based on the above described interface, the framework of the prototype system for mold cooling design optimization can be constructed, as shown in Fig. 2. First, the population is randomly initialized, where each individual (or chromosome) is represented by the binary or real value of the set of all selected design variables (see Fig. 3). In the presented case study, real representation is used. Then, each individual is evaluated by C-MOLD shrinkage and warpage analysis. Based on the simulation results the deformation of the part is computed for that chromosome. Then, to each individual is assigned a single value identifying its performance on the process (fitness). A Multi-Objective approach, described in details elsewhere (Gaspar-Cunha and Covas, 2004), is used to calculate the fitness of each individual. If the convergence objective is not satisfied (e.g., a pre-defined number of generations), the population is subjected to the operators of selection (i. e., the choice of the best individuals for crossover and/or mutation), crossover and mutation in order to obtain new individuals for the next generation/iteration (Goldberg, 1989; Coello, 2002 and Gaspar-Cunha, 2009).

In order to illustrate how the EAs work we will use a small example showing the application of selection, crossover and mutation operators (Gaspar-Cunha, 2009). The selection defines which of the individuals of the present population will act as parents of the next generation. This selection is based on the value of the global objective function determined for

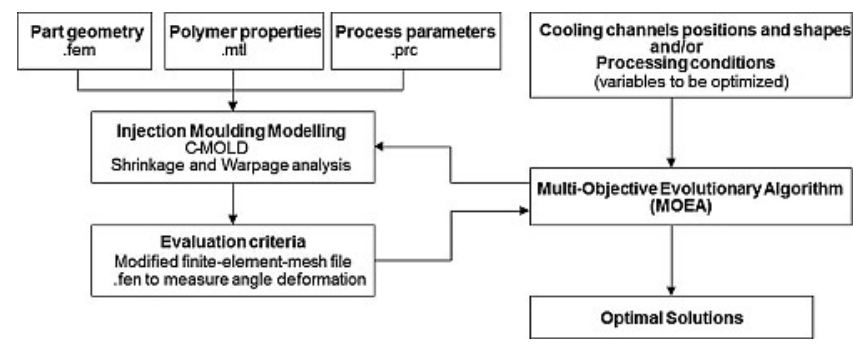

Fig. 1. Interfacing optimization routine and C-MOLD software 


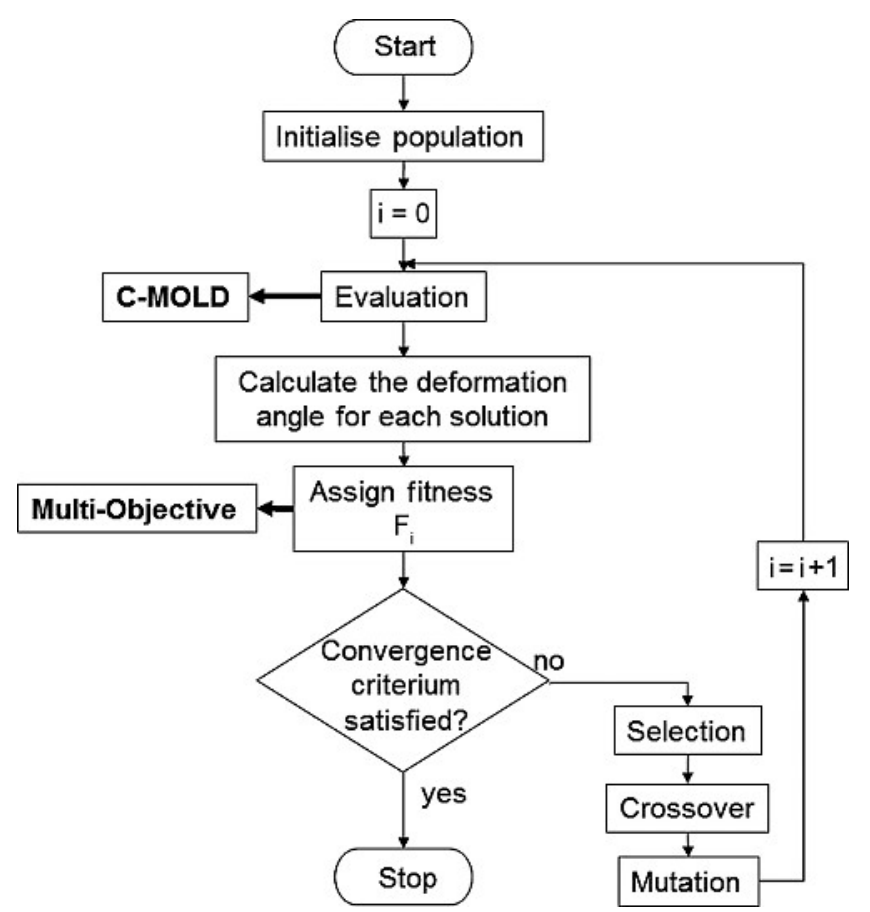

Fig. 2. Flowchart of the MOEA applied for the optimization of the cooling system design

\begin{tabular}{|c|c|c|c|c|c|c|}
\hline$y_{j}$ & $z_{j}$ & d & $T_{\mathrm{inj}}$ & $T_{w}$ & $P_{h}$ & $t_{i n j}$ \\
\hline \begin{tabular}{|l|l|l|l}
1 & 0 & 0 & 1 \\
\end{tabular} & \begin{tabular}{|l|l|l}
0 & 0 & 1 \\
\end{tabular} & \begin{tabular}{l|l|l}
1 & 1 & 1
\end{tabular} & \begin{tabular}{l|l|l}
1 & 0 & 1 \\
\end{tabular} & \begin{tabular}{l|l|l|l}
1 & 1 & 0 & 0 \\
\end{tabular} & \begin{tabular}{|l|l|l|l}
0 & 1 & 1 & 0
\end{tabular} & \begin{tabular}{|l|l|l|}
0 & 1 & 0
\end{tabular} \\
\hline
\end{tabular}

Fig. 3. Chromosome representation

each individual. Different selection schemes exist, however they are all based on the concept of giving more opportunity to the individuals (or chromosomes) with higher fitness of being selected (Goldberg, 1989 and Gaspar-Cunha, 2009).

The crossover allows the algorithm to produce new individuals for the next generation. For example, if two individuals represented in binary code (7 bits each) are selected for crossover, individual 1 [1010111] and individual 2 [1000011] and the crossover is to be applied in the position 3. Then, two new offspring are generated by exchanging the information between the parents as illustrated next: offspring 1 [101/0011] and offspring 2 [100|0111], where the sign "I" represents here the crossover point. These new individuals can be or not inserted in the new population depending if they improve or not the value of the objective function, respectively. The crossover is applied with a given rate, with means that only a percentage of the new population will be generated by crossover. Finally, the mutation consists in changing a single bit in a selected parent with a very low rate in order to obtain an offspring that will be incorporated in the new population.

\subsection{Objective Function}

Essentially, the optimization problem in the present investigation is to minimize the warpage of the part measured by the deformation angle. In the present study warpage is quantified using two conflicting objectives as described below. First, twenty four coordinates in total were taken through the C-MOLD model (Fig. 4), near of the specific points located at $5,15,25$ and $35 \mathrm{~mm}$ from the right side of the plate (as shown in Fig. 4), before and after part molding. Then the equations of eight planes defined by the specified points (Fig. 4) are computed:

$a x+b y+c z+d=0$,

where $\vec{n}=(a, b, c) \in \mathbb{N}^{3}\{(0,0,0)\}$ is the normal vector to the plane and $d$ is a real number. The corresponding angles between planes (Fig. 4) were calculated by:

$\cos \theta_{i}=\frac{\left|\mathrm{n}_{1} \cdot \mathrm{n}_{\mathrm{n}}\right|}{\left\|\mathrm{n}_{1}|| \times\right\| \mathrm{n}_{2} \|}, \mathrm{i}=1, \ldots 4$,

where $\mathrm{n}_{1}$ and $\mathrm{n}_{2}$ are normal vectors to the planes 1 and 2, respectively. The angle of the cavity molding is 28.227 degrees. After molding, the following two objectives with respect to the deformation angle deformation were defined:

i) Minimize dispersion $\sigma_{1}$ of angular measurements after molding relatively to the angle of the cavity molding (28.227 degrees):

$\min \sqrt{\frac{\sum_{i=1}^{4}\left(28.227-\theta_{i}\right)^{2}}{3}}$.

ii) Minimize dispersion $\sigma_{2}$ of differences $\mathrm{d} \theta_{\mathrm{i}}=28.227$ $\theta_{\mathrm{i}}, \mathrm{I}=1, \ldots, 4$, relatively to their mean value $\mathrm{d} \bar{\theta}_{\mathrm{i}}$ :

$\min \sqrt{\frac{\sum_{\mathrm{i}=1}^{4}\left(\mathrm{~d} \theta_{\mathrm{i}}-\overline{\mathrm{d} \theta_{\mathrm{i}}}\right)^{2}}{3}}$ where $\overline{\mathrm{d} \theta_{\mathrm{i}}}=\frac{\sum_{\mathrm{i}=1}^{4} \mathrm{~d} \theta_{\mathrm{i}}}{4}$.

The goal of each objective is:

i) to obtain a part with an angle closer as much to 28.227 , after molding (angle warpage);

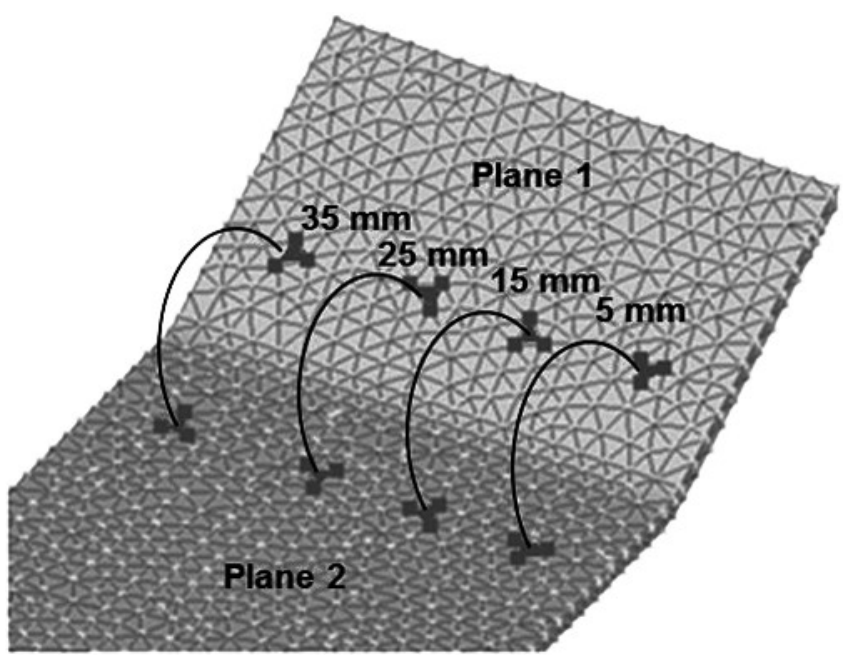

Fig. 4. Coordinates and angles between planes on C-MOLD model (before and after molding) 
ii) to maintain unchanged the differences $\mathrm{d} \theta_{\mathrm{i}}, \mathrm{I}=1, \ldots, 4$, along the specific points $(5,15,25$ and $35 \mathrm{~mm})$ in order to minimize the effect of plane warpage.

These two objectives are conflicting and will be optimized simultaneously. Concerning the modelling programme, the geometric and process constraints considered were:

- geometric constraints:

- upper/lower-bound constraints on the coordinates of the cooling channels;

- limits on the distance between the cooling channels;

- limits on the distance between each channel and the cavity boundary.

- process restrictions:

- the molding has to be completely filled, obviously no short-shots were admitted;

- the computed values of the maximum shear stress and strain-rate were limited to their critical values (defined on the C-MOLD database) in order to avoid other potential defects (e. g., shark skin).

\section{Case Study}

The cooling system considered in this investigation uses cylindrical cooling channels and water as coolant fluid. The geometry is a rectangular L-shape molding with a curved end as shown in Fig. 5. The molding has the following nominal dimensions: $1.5 \mathrm{~mm}$ of thickness, $40 \mathrm{~mm}$ of width and $134 \mathrm{~mm}$ of length. The finite element mesh has 874 triangular elements. The initial cooling system layout is presented in Fig. 5.

The part is molded in polystyrene (Styron 678E, Bayer). Table 1 gives a summary of the relevant polymer properties used for the flow simulations (C-MOLD database).

Mold material selected is P20 steel and concerning the processing conditions two cases were considered, one where they are constant and other where they are optimized. The simulations in C-MOLD are based on a hybrid finite-element/finitedifference/control-volume numerical solution of the generalized Hele-Shaw flow equation of a compressible viscous fluid under non-isothermal conditions. The polymer rheological and PVT description were modelled by a Cross-WLF and the Tait modified equations, respectively. More details about the software are described in related literature (Hieber and Shen, 1980; Chiang et al., 1991a, 1991b and Viana, 1999). The simulations considered the C-MOLD integrated shrinkage \& warpage analysis that includes polymer melt flow and mold cooling analyses, residual stress calculations, and structural analysis.

The cooling system was modelled by sixteen coordinates describing the locations of the two cooling channels, one in each mold side. Each location is defined by the $\mathrm{y}$ and $\mathrm{z}$ coordinates of the cooling line centre in the $y-z$ plane and by the $x$ coordinate describing the depth of the cooling line along the $x$ axis ( $\mathrm{x}$ is maintained constant along the optimization process; see Fig. 5). Another selected design variable is the cooling channel diameter. Four processing variables were also selected, namely, the injection time, the melt and mold temperatures and the holding pressure, based on their potential relevance on the part quality. Table 2 resumes the selected design variables and their corresponding values ranges.

The RPSGAe uses a real representation of the variables, a simulated binary crossover, a polynomial mutation and a roulette wheel selection strategy (Gaspar-Cunha and Covas, 2004; Goldberg, 1989; Coello, 2002 and Deb, 2001). The RPSGAe was applied using the following parameters: 50 generations

\begin{tabular}{|c|c|}
\hline Property & Value unit \\
\hline Melt density & $968.6 \mathrm{~kg} / \mathrm{m}^{3}$ \\
Typical melt temperature & $503 / 230 \mathrm{~K} /{ }^{\circ} \mathrm{C}$ \\
Maximum shear stress & $2.4 \times 10^{5} \mathrm{~Pa}$ \\
Maximum shear rate & $4 \times 10^{4} 1 / \mathrm{s}$ \\
Specific heat & $2100 \mathrm{~J} / \mathrm{kg} \mathrm{K}$ \\
Thermal conductivity & $0.15 \mathrm{~W} / \mathrm{m} \mathrm{K}$ \\
\hline
\end{tabular}

Table 1. Typical properties of the used polystyrene

\begin{tabular}{|c|c|}
\hline Design variable & Value range \\
\hline $\mathrm{Y}$ coordinate $(\mathrm{mm})-\mathrm{y}_{1}, \ldots, \mathrm{y}_{16}$ & {$[0,160]$ subject to constraint } \\
$\mathrm{Z}$ coordinate $(\mathrm{mm})-\mathrm{z}_{1}, \ldots, \mathrm{z}_{16}$ & {$[-20,50]$ subject to constraint } \\
Cooling channel diameter $(\mathrm{mm})-\mathrm{d}$ & {$[5,10]$} \\
Injection time $(\mathrm{s})-\mathrm{t}_{\mathrm{inj}}$ & {$[0.5,3]$} \\
Melt temperature $\left({ }^{\circ} \mathrm{C}\right)-\mathrm{T}_{\mathrm{inj}}$ & {$[180,280]$} \\
Mold temperature $\left(\mathrm{T}_{\mathrm{w}}\right.$ & {$[30,70]$} \\
Holding Pressure $(\%$ of maximum & {$[7,38]$} \\
machine injection pressure $)$ & \\
& \\
\hline
\end{tabular}

Table 2. Design and processing variables and their value ranges

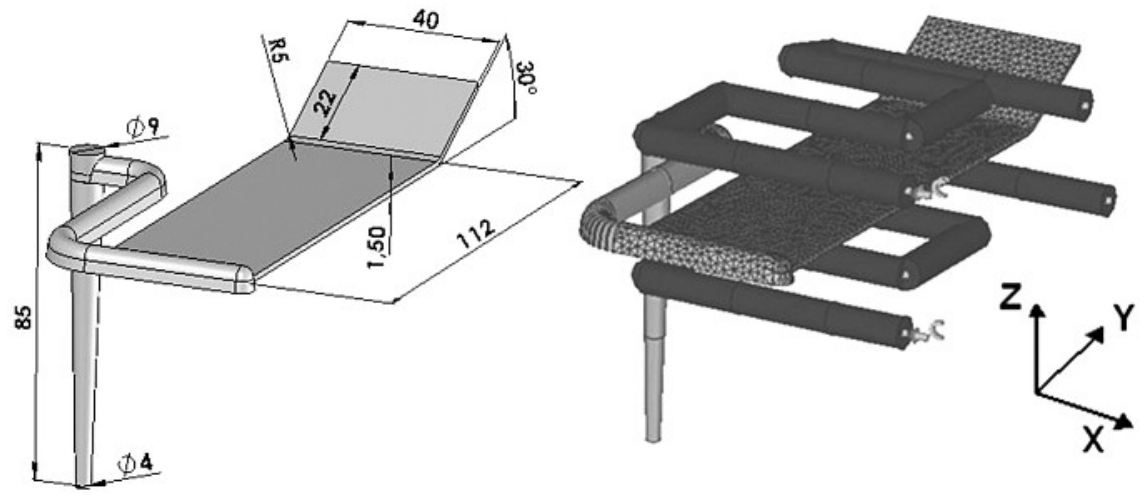

Fig. 5. Molding geometry, mesh and initial cooling system (dimensions in $\mathrm{mm}$ ) 
(or iterations), crossover rate of 0.8 , mutation rate of 0.05 , internal and external populations with 200 individuals, limits of the clustering algorithm set at 0.2 and $\mathrm{N}_{\text {Ranks }}$ at 30 . These values resulted from a carefully analysis made in a previous work (Gaspar-Cunha and Covas, 2004).

The proposed optimization methodology will be used for setting the diameter and coordinates of the cooling channels and/or to define the selected processing conditions.

\section{Results}

Three different studies were performed. First, an analysis of variance (ANOVA and MANOVA) was made considering simulation results with the aim to check if the parameters considered are statistically significant for the objectives used. This ANOVA/MANOVA analysis only was made for comparison purposes and is not necessary in the optimization scheme based on EAs proposed. Then, the RPSGAe algorithm was used to optimize the process considering two different situations, one considering the operating conditions constant and other where these conditions are optimized simultaneously with cooling channels design.

At this point is important to clarify that the computation time for an optimization run only depends on the number of times the C-MOLD software is called. In the present case is possible to get a complete optimization in circa of 10 hours in a personal computer with an Intel i7 processor at 2.67 MHz. An optimal solution is obtained after a RPSGA optimization runs (where the C-MOLD software is called hundreds of times).

\subsection{Analysis of Modelling Results}

The molding program includes changes of the injection time, melt and mold temperatures and holding pressure, in two levels (Table 2) according to design of analytical simulations plan. A total of 16 different moldings were obtained, as listed in Table 3.

The effect of the processing conditions on the angular measurements after molding is listed in Table 4.

Two types of analysis were performed with these simulation results, namely an Analysis of Variance (ANOVA) and a Multivariate Analysis of Variance (MANOVA) (Chatfield and Collins, 1996). In the former, the analysis is performed on each of the dependent variables. In the MANOVA analysis the four variables are considered simultaneously in order to detect a potential degree of correlation between them.

Table 5 presents the results for the responses of the analytical modeling package. The table lists the significant terms (5\% level) for the multivariate (MANOVA) and univariate (ANOVA) analysis. These results were obtained using the SPSS software considering different multivariate tests (Pillai's trace, Wilk's Lambda, Hotteling's trace and Roy's Largest Root).

According to MANOVA, all the main effects are statistically significant and only the two-way interaction between $T_{i n j}$ and $\mathrm{Ph}$ is statistically significant. However, when the effects of variables are considered individually, one can conclude that injection time is not important on dispersion $\sigma_{2}$ of differences $\mathrm{dq}_{\mathrm{i}},=28.2-\mathrm{q}_{\mathrm{i}}, \mathrm{I}=1, \ldots, 4$, and mold temperature is not important

\begin{tabular}{|c|c|c|c|c|}
\hline Run & $\begin{array}{c}\mathrm{t}_{\text {inj }} \\
\mathrm{S}\end{array}$ & $\begin{array}{c}\mathrm{T}_{\mathrm{inj}} \\
{ }^{\circ} \mathrm{C}\end{array}$ & $\begin{array}{c}\mathrm{T}_{\mathrm{w}} \\
{ }^{\circ} \mathrm{C}\end{array}$ & $\begin{array}{r}\mathrm{Ph} \\
\%\end{array}$ \\
\hline 1 & 0.5 & 180 & 30 & 7 \\
2 & 0.5 & 180 & 30 & 38 \\
3 & 0.5 & 180 & 70 & 7 \\
4 & 0.5 & 180 & 70 & 38 \\
5 & 0.5 & 280 & 30 & 7 \\
6 & 0.5 & 280 & 30 & 38 \\
7 & 0.5 & 280 & 70 & 7 \\
8 & 0.5 & 280 & 70 & 38 \\
9 & 3 & 180 & 30 & 7 \\
10 & 3 & 180 & 30 & 38 \\
11 & 3 & 180 & 70 & 7 \\
12 & 3 & 180 & 70 & 38 \\
13 & 3 & 280 & 30 & 7 \\
14 & 3 & 280 & 30 & 38 \\
15 & 3 & 280 & 70 & 7 \\
16 & 3 & 280 & 70 & 38 \\
\hline
\end{tabular}

Table 3. Design of analytical simulations (independent variables)

\begin{tabular}{|c|c|c|}
\hline Run & $\sigma_{1}$ & $\sigma_{2}$ \\
\hline 1 & 0.01876 & 0.00136 \\
2 & 0.02614 & 0.00387 \\
3 & 0.00851 & 0.00227 \\
4 & 0.02456 & 0.00733 \\
5 & 0.02696 & 0.00140 \\
6 & 0.05494 & 0.00680 \\
7 & 0.01914 & 0.00184 \\
8 & 0.06589 & 0.01293 \\
9 & 0.01599 & 0.00179 \\
10 & 0.02178 & 0.00556 \\
11 & 0.00956 & 0.00220 \\
12 & 0.01989 & 0.00706 \\
13 & 0.02058 & 0.00076 \\
14 & 0.03960 & 0.00797 \\
15 & 0.01274 & 0.00169 \\
16 & 0.05391 & 0.01505 \\
\hline
\end{tabular}

Table 4. Angular measurements resulting from variation of the processing conditions (dependent variables)

\begin{tabular}{|c|c|c|c|}
\hline \multirow{2}{*}{ Effect } & \multirow{2}{*}{ Multivariate analysis } & \multicolumn{2}{|c|}{ Univariate analysis } \\
\cline { 3 - 4 } & & $\sigma_{1}$ & $\sigma_{2}$ \\
\cline { 3 - 4 } & & $*$ & $*$ \\
\hline Intercept & $*$ & $*$ & - \\
$\mathrm{t}_{\text {inj }}$ & $*$ & $*$ & $*$ \\
$\mathrm{~T}_{\text {inj }}$ & $*$ & - & $*$ \\
$\mathrm{~T}_{\mathrm{w}}$ & $*$ & $*$ & $*$ \\
$\mathrm{Ph}_{\mathrm{inj}} * \mathrm{~T}_{\mathrm{inj}}$ & $*$ & - & - \\
$\mathrm{t}_{\mathrm{inj}} * \mathrm{~T}_{\mathrm{w}}$ & - & - & - \\
$\mathrm{t}_{\mathrm{inj}} * \mathrm{Ph}$ & - & - & - \\
$\mathrm{T}_{\mathrm{inj}} * \mathrm{~T}_{\mathrm{w}}$ & - & - & - \\
$\mathrm{T}_{\mathrm{inj}} * \mathrm{Ph}$ & - & $*$ & $*$ \\
$\mathrm{~T}_{\mathrm{w}} * \mathrm{Ph}$ & $*$ & $*$ & $*$ \\
\hline
\end{tabular}

(* statistically significant, - statistically non-significant)

Table 5. Significant processing factors from ANOVA and MANOVA analysis 
on dispersion $\sigma_{1}$ of angular measurements after molding. Also, the two-way interactions between $\mathrm{T}_{\mathrm{inj}} * \mathrm{Ph}$ and $\mathrm{T}_{\mathrm{w}} * \mathrm{Ph}$ are statistically significant.

Figure 6 shows the effects of some interactions between factors $\left(\mathrm{t}_{\mathrm{inj}} * \mathrm{~T}_{\mathrm{inj}}, \mathrm{t}_{\mathrm{inj}} * \mathrm{~T}_{\mathrm{W}}, \mathrm{t}_{\mathrm{inj}} * \mathrm{Ph}\right)$ on the dispersion coefficients $\sigma_{1}$ and $\sigma_{2}$. In general, the values of $\sigma_{1}$ decreases with injection time and the values of $\sigma_{2}$ increase with it except when holding pressure equals $7 \%$. The values of $\sigma_{1}$ are lower when melt temperature and holding pressure decreases and mold temperature increases. $\sigma_{2}$ values are lower for lower values of all shown factors.

The main conclusion from this analysis is that there is an interaction between both objectives considered $\sigma_{1}$ and $\sigma_{2}$, i.e., is not indifferent to consider these objectives separately or simultaneously.

\subsection{Processing Conditions Optimization Results}

Firstly, the optimization methodology above proposed was used for setting the processing conditions of the case study molding in order to accomplish the objectives given by Eqs. 3 and 4 , i.e., to minimize the part warpage and distortion. The results obtained in the objectives space are shown in Fig. 7 and the associated processing conditions for points $\mathrm{P} 1$ to $\mathrm{P} 7$ are shown in Table 6.

From Fig. 7 can be observed that points P1, P2 and P3 (open symbols), that belong to the initial population, have significantly higher holding pressure values than points P4, P5, P6 and P7 that belong to the final population (filled symbols). This fact indicates that to minimize the deformation angle and distortion a lower holding pressure must be applied. Points P4

\begin{tabular}{|c|c|c|c|c|c|c|}
\hline & \multicolumn{3}{|c|}{ Variables } & \multicolumn{2}{c|}{ Objectives } \\
\hline $\begin{array}{c}\text { Solu- } \\
\text { tions }\end{array}$ & $\begin{array}{c}\mathrm{t}_{\text {inj }} \\
\mathrm{s}\end{array}$ & $\begin{array}{c}\mathrm{T}_{\mathrm{inj}} \\
{ }^{\circ} \mathrm{C}\end{array}$ & $\begin{array}{c}\mathrm{T}_{\mathrm{w}} \\
{ }^{\circ} \mathrm{C}\end{array}$ & $\begin{array}{c}\mathrm{P}_{\mathrm{h}} \\
\%\end{array}$ & $\sigma_{1}$ & $\sigma_{2}$ \\
\hline${ }^{\circ}$ & & & & \\
\hline P1 & 1.21 & 265 & 70 & 35.1 & 0.04762 & 0.01001 \\
P2 & 2.87 & 225 & 67 & 34.9 & 0.02998 & 0.00802 \\
P3 & 2.43 & 225 & 65 & 21.9 & 0.01866 & 0.00353 \\
P4 & 2.65 & 226 & 56 & 8.1 & 0.00169 & 0.00126 \\
P5 & 2.60 & 229 & 54 & 7.9 & 0.00275 & 0.00051 \\
P6 & 2.64 & 222 & 69 & 7.1 & 0.01156 & 0.00034 \\
P7 & 2.53 & 229 & 65 & 7,3 & 0.01281 & 0.00026 \\
\hline
\end{tabular}

Table 6. Optimal processing conditions to minimize the deformation angle and plane distortion of the moulding

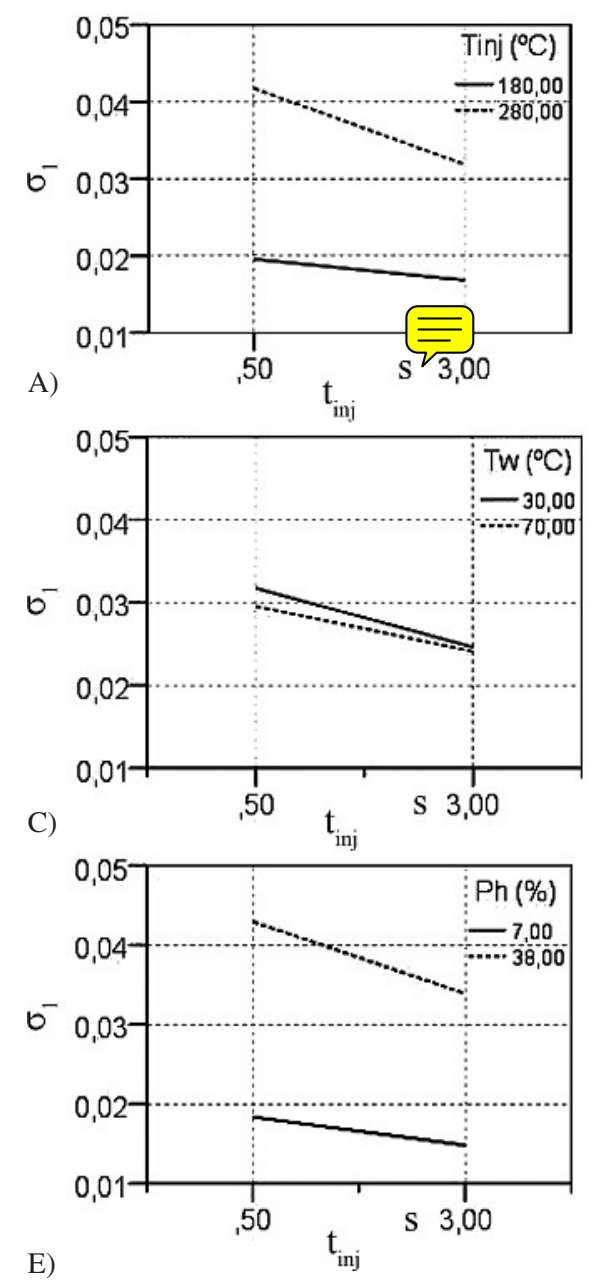

6
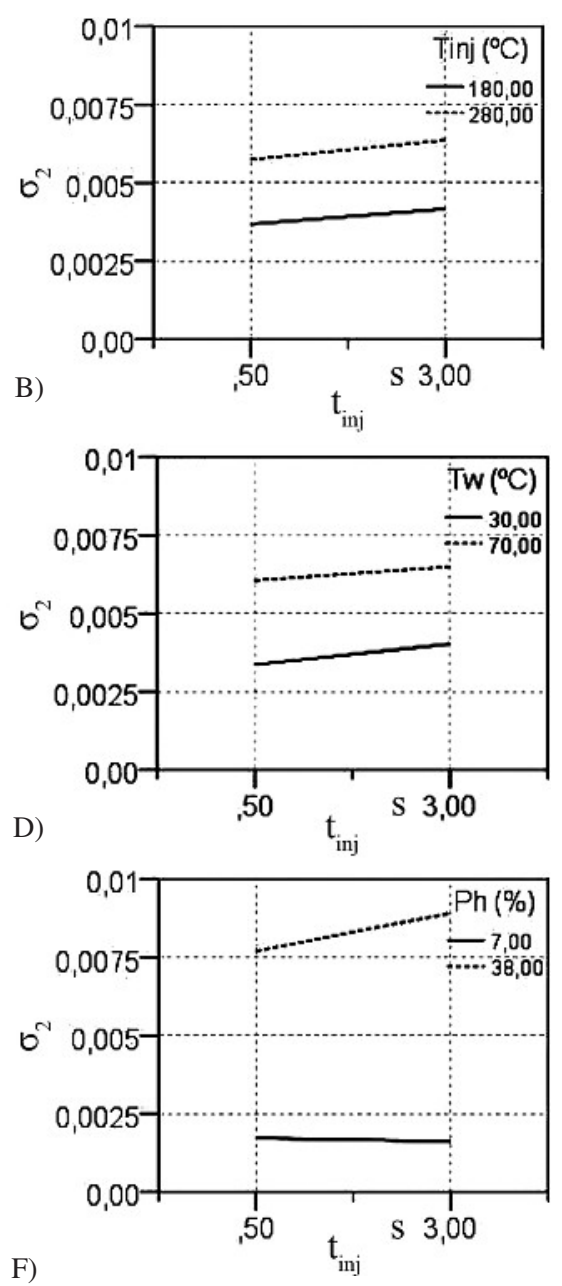

Fig. 6. Effect graphs for dispersion one and two vs. injection time for different levels of $T_{i n j}, T_{W}$ and $P h$ 


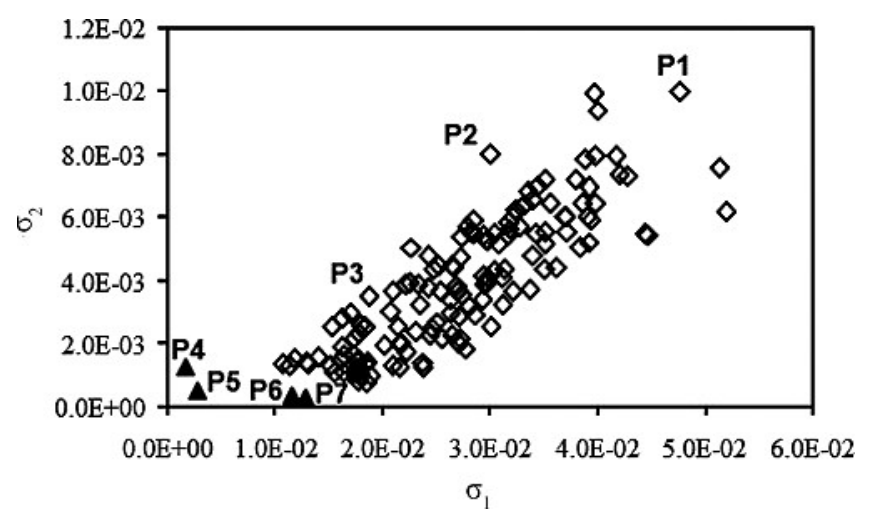

Fig. 7. Processing conditions optimization results. Full symbols: Pareto frontier at $50^{\text {th }}$ generation; open symbols: initial population $\left(\sigma_{1}\right.$ : dispersion of angular measurements after molding, $\sigma_{2}$ : dispersion of differences $d \theta_{i}, i=1, \ldots 4$, , relatively to their mean value $\frac{d \theta_{i}}{2}$

and P5 are the solutions with minimal deformation angle (i.e. higher minimization of $\sigma_{1}$ ) and points P6 and P7 represent the solutions with minimal part distortion (i.e. higher minimization of $\sigma_{2}$ ).

Analysing Table 6, it is clear that injection time and melt temperature values are very similar in all solutions (except for P1), but mold temperatures of solutions P4 and P5 are lower than the mold temperatures of solutions P6 and P7; and holding pressures of solutions P1 to P3 are higher than holding pressures of solutions $\mathrm{P} 4$ to $\mathrm{P} 7$. Thus, solutions with injection times on [2.45 to $2.87 \mathrm{~s}$ ], melt temperatures on [222 to $229^{\circ} \mathrm{C}$ ], mold temperature of 54 to $56^{\circ} \mathrm{C}$ and holding pressure equal to 7 to 9.8.1\% results in a molding with the lowest deformation angle and the solutions with mold temperature of 65 to $70^{\circ} \mathrm{C}$ and holding pressure of 22 to $35 \%$ leads to a lower part distortion.

These results are in accordance with the statistical analysis done in the previous section. The best results (i.e., that minimize simultaneously both objectives) are achieved for lower holding pressure, intermediate mold and injection temperatures and higher injection times.

\subsection{Cooling Channels Design Optimization Results}

Secondly, the proposed optimization methodology is applied to find the best cooling channels design variables. In this optimization procedure, the processing conditions were maintained constant, according to Table 7 . To measure the optimality of the solutions, the objectives given by Eqs. 3 and 4 were again used.

\begin{tabular}{|c|c|}
\hline Parameter & Value unit \\
\hline Injection temperature & $230^{\circ} \mathrm{C}$ \\
Mold temperature & $50^{\circ} \mathrm{C}$ \\
Holding pressure & $\begin{array}{c}17 \% \text { of the maximum machine } \\
\text { injection pressure } \\
\text { Holding time } \\
15 \mathrm{~s}\end{array}$ \\
Injection time \\
$0.5 \mathrm{~s}$ \\
\hline
\end{tabular}

Table 7. Processing conditions used in the simulations
This study was divided in two parts: firstly, the simulations consider a cold runners system; second, the simulations consider a direct hot runner.

\subsubsection{Optimization with Cold Runners}

The results obtained in the criteria's space for the initial and final generations are presented in Fig. 8 and the cooling channels locations of points $\mathrm{P} 1$ to $\mathrm{P} 6$ are shown in Fig. 9.

As an example, points P1 and P2 (belonging to the initial population) were picked in Fig. 8, in order to study their evolution along the optimization process. The cooling channels designs of these points are, also, represented in Fig. 9. The evolution of point $\mathrm{P} 1$ trough the optimization process originates solutions represented by point P3, P4 and P5 in the final population and point $\mathrm{P} 2$ originates solution $\mathrm{P} 6$ in final population. Cooling channels locations represented on Fig. 9 (points P3, P4 and P5 in Fig. 8, respectively) provide a higher minimization of $\sigma_{1}$, and cooling channel location of Fig. 9 (point P6 in Fig. 8) assure a higher minimization of $\sigma_{2}$. Points P3, P4 and P5 have cooling channels designs with similar shape. On the other hand, point P6 has a cooling channel design with a different shape. This means that solutions of points P3, P4 and P5 are more efficient to obtain a polymeric part with an angle similar to $28.2^{\circ}$ after processing, i.e., lower deforma-

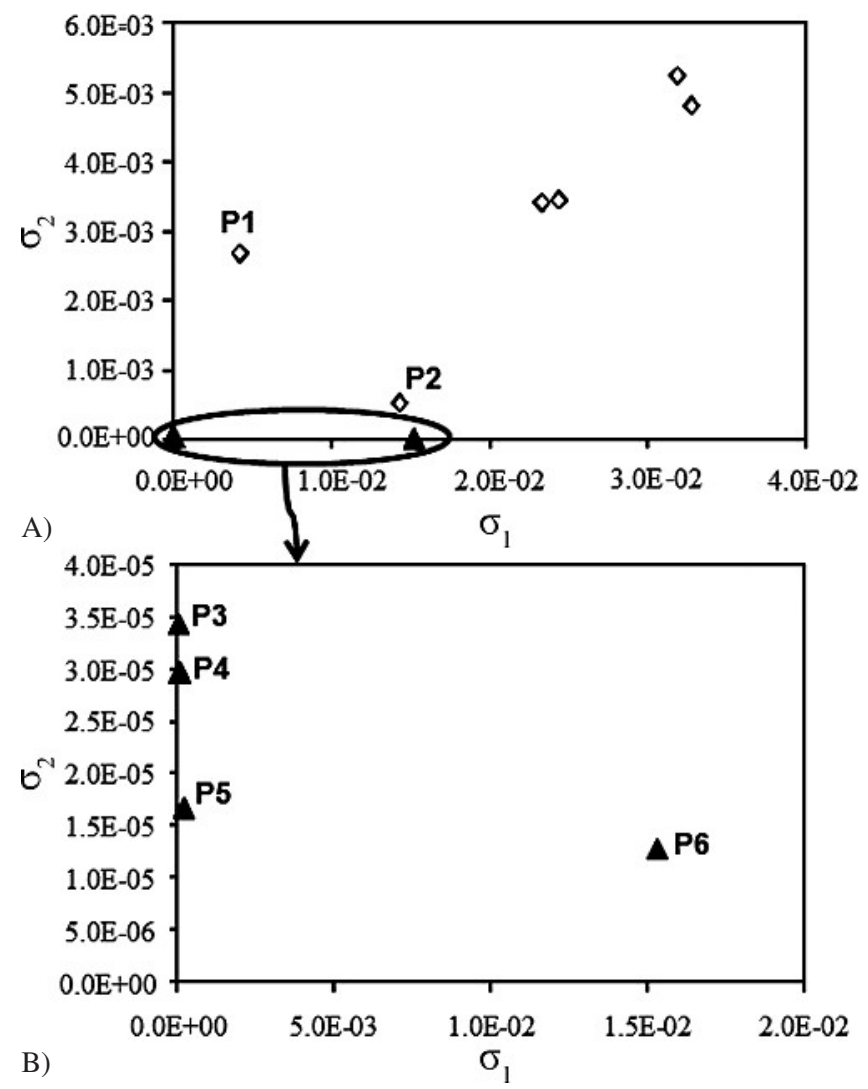

Fig. 8. Cooling channels design optimization results: (A) initial population and $(B)$ Pareto frontier at $50^{\text {th }}$ generation $\left(\sigma_{1}\right.$ : dispersion of angular measurements after molding, $\sigma_{2}$ : dispersion of differences $d \theta_{i}, i=1, \ldots 4$, relatively to their mean value $\left.\frac{d \theta_{i}}{}\right)$

Intern. Polymer Processing XXVII (2012) 2 

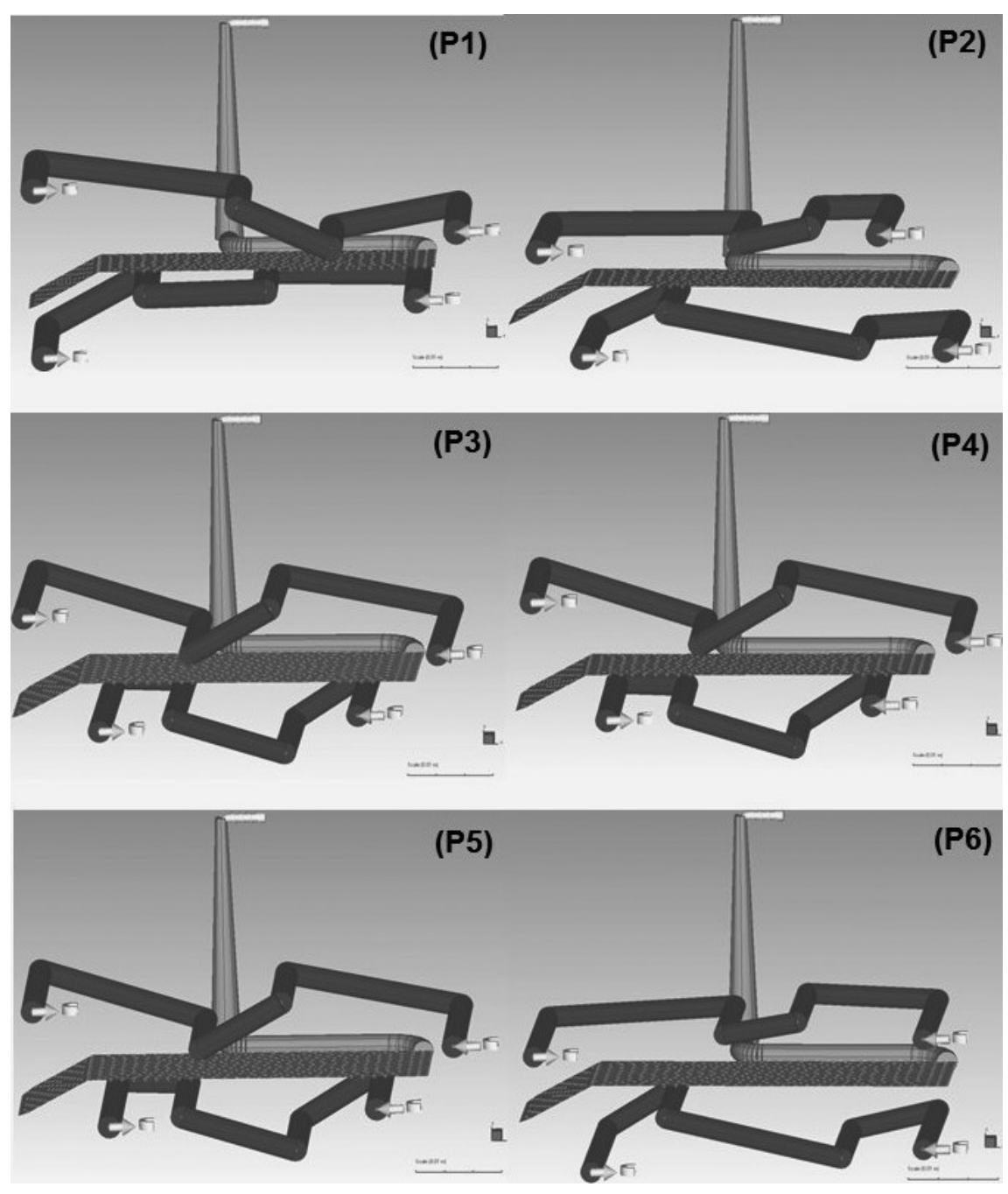

Fig. 9. Optimal cooling channels designs: solutions corresponding to points P1 to P6 represented in Fig. 8 tion angle, and the solution of point P6 is more indicated to minimize the effect of distortion of the part because it is better in maintaining unchanged the difference $\mathrm{d} \theta_{\mathrm{i}}, \mathrm{i}=1, \ldots, 4$, along the width of the part. It is also important to note that the cooling channel diameter of solutions who guarantee a higher minimization of $\sigma_{1}$ is of $8 \mathrm{~mm}$, and cooling channel diameter of solution that assure a higher minimization of $\sigma_{2}$ is of $7 \mathrm{~mm}$.

\subsubsection{Optimization with Direct Hot Runner}

The results obtained in the criteria's space for the initial and final generations are presented in Fig. 10 and the cooling channels locations of points P1 to P4 are shown in Fig. 11.

Similar to what have done previously, points P1 and P2 (belonging to initial population) were picked in Fig. 10 to study their evolution along the optimization process. The cooling channels designs of these points are represented in Fig. 11. Both points of the final population P3 and P4 have similar cooling channels layouts, and the criteria's values determined for these two solutions are very similar too. This means that these two solutions can be considered as only one solution. Therefore, our design cooling channel optimization problem with hot runner system have a unique solution, with a cooling channel diameter of $7 \mathrm{~mm}$.

\subsection{Simultaneous Processing Conditions and Cooling Channels Design Optimization Results}

Finally, the proposed optimization methodology was used to find simultaneously the best cooling channels design and processing conditions that minimize the deformation angle and the part warpage. In this case only cold runners were used. The results obtained in the criteria's space for the initial and final generations are presented in Fig. 12.

The cooling channels locations of points P1 to P5 are shown in Fig. 13 and the correspondent processing conditions are listed in Table 8. The final generation solutions (P3, P4 and P5) feature cooling channels designs with identical layout sand the optimal processing conditions values are also very similar. Namely, the injection time should be settled at $2.52 \mathrm{~s}$, the melt and mold temperatures at $241^{\circ} \mathrm{C}$ and $32^{\circ} \mathrm{C}$ respectively, and 


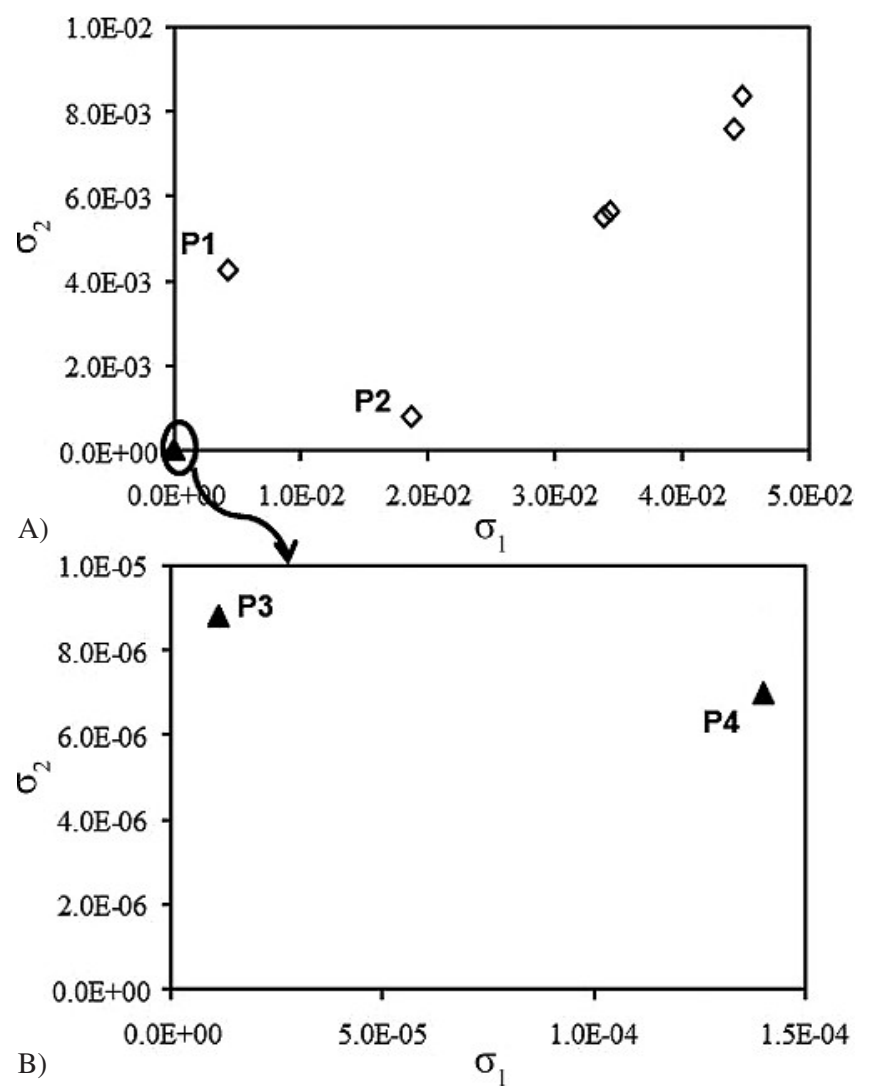

Fig. 10. Cooling channels design optimization results: (A) initial population and $(B)$ Pareto frontier at $50^{\text {th }}$ generation $\left(\sigma_{1}\right.$ : dispersion of angular measurements after molding, $\sigma_{2}$ : dispersion of differences $d \theta_{i}, i=1, \ldots 4$, relatively to their mean value $\left.\frac{\theta}{d \theta_{i}}\right)$

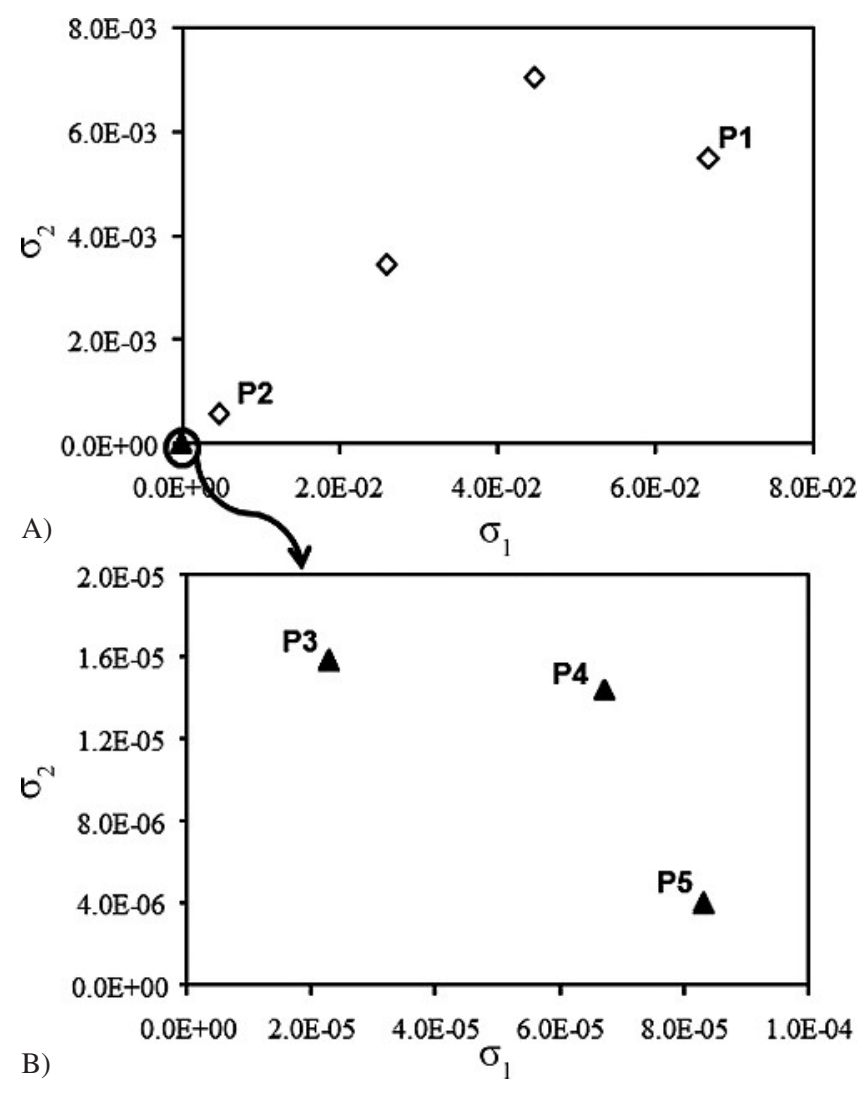

Fig. 12. Processing conditions and cooling channels design optimization results: (A) Initial population and Pareto frontier at $50^{\text {th }}$ generation; $(B)$ Pareto frontier at $50^{\text {th }}$ generation. $\left(\sigma_{1}\right.$ : dispersion of angular measurements after molding, $\sigma_{2}$ : dispersion of differences $d \theta_{i}, i=1, \ldots 4$, relatively to their mean value $\left.\overline{d \theta_{i}}\right)$
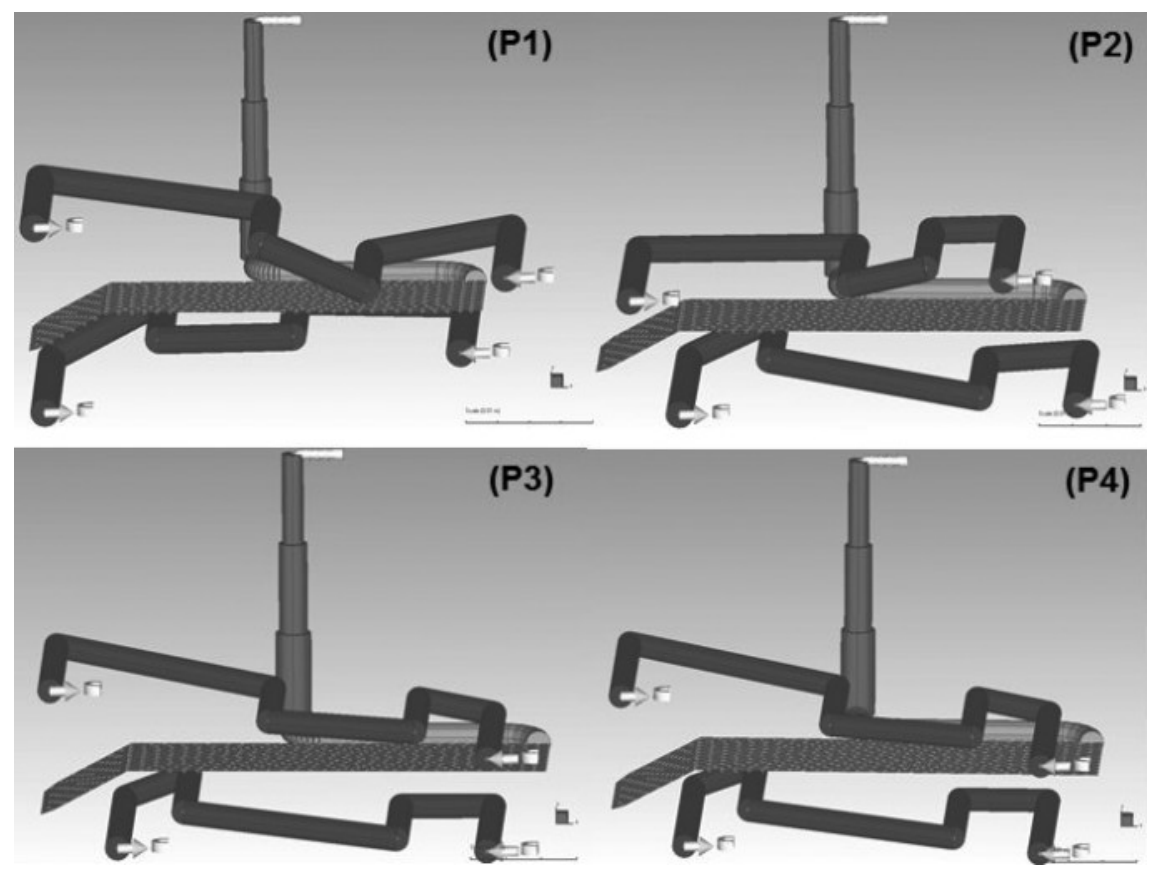

Fig. 11. Optimal cooling channels designs: solutions corresponding to points P1 to P4 represented in Fig. 9 

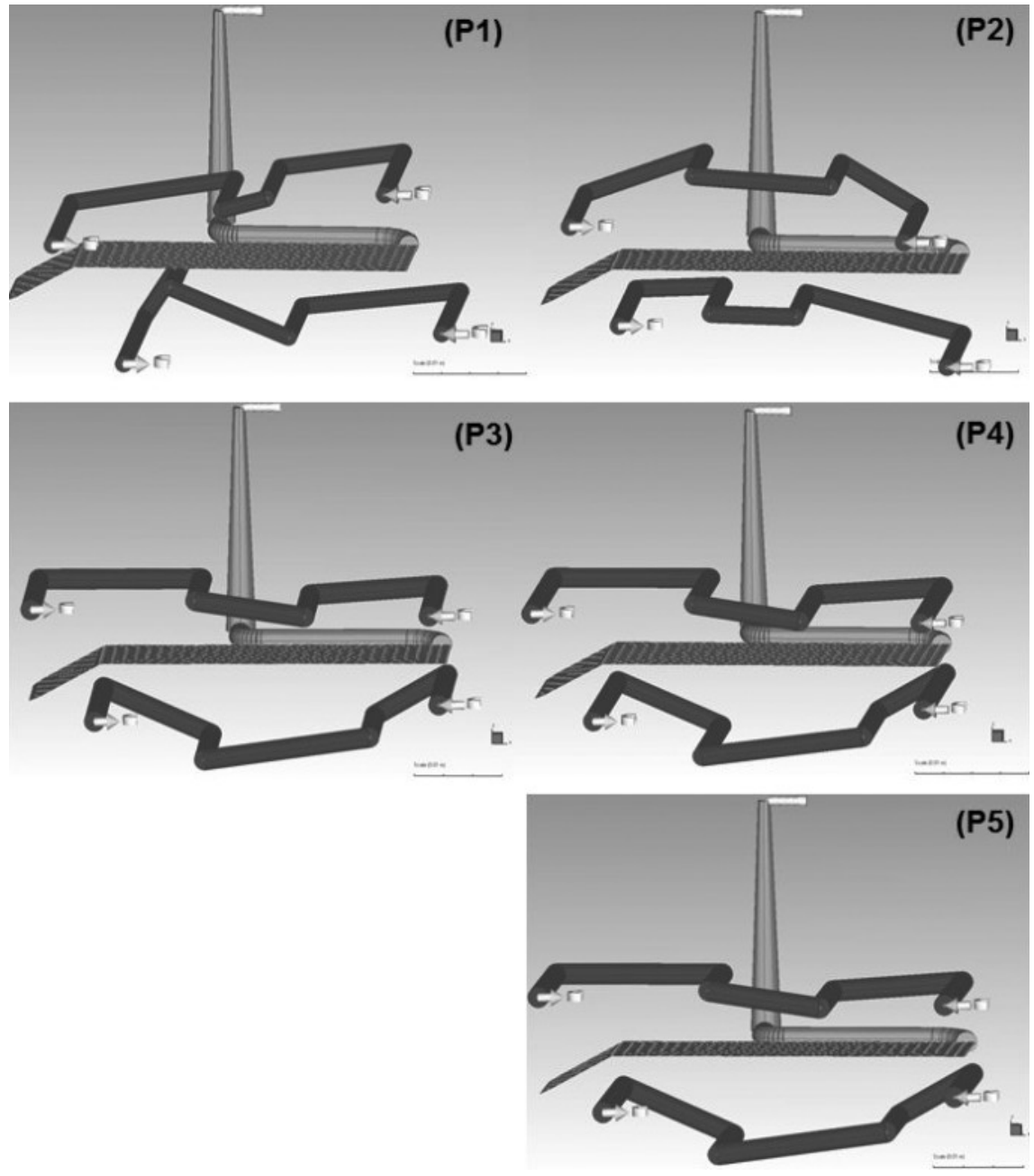

Fig. 13. Optimal cooling channels designs: solutions corresponding to points P1 to P5 represented in Fig. 11 holding pressure at $11.7 \%$ of maximum machine injection pressure.

The optimization of cooling channels layout and processing conditions must be done simultaneously.

\section{Conclusions}

In this work, a multi-objective optimization methodology based on Evolutionary Algorithms (MOEA) was applied to the optimization of processing conditions and cooling channels locations of a L-shaped rectangular molding in order to minimize the effect of part warpage and deformation angle.

Initially, analysis of variance (ANOVA and MANOVA) are performed allowed to establish the set of iterations between the factors studied (i.e., the iterations between the objectives to be accomplished and the decision variables). As expected, it is not indifferent to consider the objectives separately or simultaneously. This shows the need of an optimization methodology able to take into account both objectives simultaneously such as the one proposed here.

The optimization methodology used was able to produce results with physical meaning for three different studies: first, for individual optimization of processing conditions, then for individual optimization of cooling channels locations and finally simultaneously optimization of processing conditions and cooling channels locations.

The best strategy is to optimize simultaneously the cooling channel layout and processing conditions.

\section{References}

Chatfield, C., Collins, A. J.: Introduction to Multivariate Analysis, Chapman \& Hall, London (1996)

Chiang, H. H., et al., "A Unified Simulation of the Filling and Postfilling Stages in Injection Molding. Part I: Formulation", Polym. Eng. Sci., 31, 116-124 (1991), DOI:http://dx.doi.org/10.1002/pen.760310210

Chiang, H. H., et al., "A Unified Simulation of the Filling and Postfilling Stages in Injection Molding. Part II: Experimental Verification", Polym. Eng. Sci., 31, 125-139 (1991),

DOI:http://dx.doi.org/10.1002/pen.760310211

Coello Coello, C. A., et al.: Evolutionary Algorithms for Solving Multi-objective Problems, Kluwer Academic, Boston (2002)

Deb, K.: Multi-objective Optimization Using Evolutionary Algorithms, John Wiley \& Sons, New York (2001)

Duff, A. L., "Increased Speed too Market Using CAE Simulation for Injection Molded Plastics Parts", Proceedings $58^{\text {th }}$ Annual Meeting of the Society of Plastics Engineers, Orlando (2000)

Gaspar-Cunha, A., Covas, J. A., "RPSGAE - Reduced Pareto Set Genetic Algorithm: Application to Polymer Extrusion", in Lecture 
Notes in Economics and Mathematical Systems, Gandibleux, X., et al. (Eds.), Springer, Berlin, p. 221-255 (2004)

Gaspar-Cunha, A.: Modelling and Optimisation if Single Screw Extrusion - Using Multi-objective Evolutionary Algorithms, Lambert Academic, Köln, Germany (2009)

Goldberg, D. E.: Genetic Algorithms in Search, Optimization and Machine Learning, Addison-Wesley, Massachusetts (1989)

Hieber, C. A., Shen, S. F., "A Finite-Element/Finite-Difference Simulation of the Injection-Molding Filling Process", J. Non-Newtonian Fluid Mech., 7, 1-32 (1980), DOI:http://dx.doi.org/10.1016/0377-0257(80)85012-9

Lam, Y. C., "An Evolutionary Approach for Cooling System Optimization in Plastic Injection Moulding", Int. J. Prod. Res., 42, 2047 2061 (2004), DOI:http://dx.doi.org/10.1080/00207540310001622412

Mathey, E., "Automatic Optimization of the Cooling of Injection Mold Based on the Boundary Element Method", Materials Processing and Design: Modelling, Simulation and Applications匹???please provide correct abbreviation for this journal??? , 712, 222-227 (2004)

Menges, G., Mohren, P., "How to Make Injection Molds", New York: Hanser (1986)

Park, S., Kwon, T., "Thermal and Design Sensitivity Analyses for Cooling System of Injection Mold, Part 1: Thermal Analysis", J. Manuf. Sci. Eng., 120, 287-295 (1998a), DOI:http://dx.doi.org/10.1115/1.2830126

Park, S., Kwon, T., "Thermal and Design Sensitivity Analyses for Cooling System of Injection Mold, Part 2: Design Sensitivity Analysis", J. Manuf. Sci. Eng., 120, 296-305 (1998b), DOI:http://dx.doi.org/10.1115/1.2830127

Park, S., Kwon, T., "Optimal Cooling System Design for the Injection Molding Process", Polym. Eng. Sci., 38, 1450-1462 (1998c), DOI:http://dx.doi.org/10.1002/pen.10316

Pirc, N., et al., "3D BEM-Based Cooling-Channel Shape Optimization for Injection Moulding Processes", Int. J. Simul. Multi. Design Optim., 2, 245-252 (2008b) DOI:http://dx.doi.org/10.1051/ijsmdo:2008033

Pirc, N., et al., "Optimization Of BEM-Based Cooling Channels Injection Moulding Using Model Reduction", Int. J. Mater. Form., 口??volume, pages??? 1043-1046 (2008a)

Qiao, H., "A Systematic Computer-aided Approach to Cooling System Optimal Design in Plastic Injection Molding", Int. J. Mech. Sci., 48, 430-439 (2006),

DOI:http://dx.doi.org/10.1016/j.ijmecsci.2005.11.001
Tang, L., et al., "A Computer-aided Optimization Approach for the Design of Injection Mold Cooling Systems", J. Mech. Des., 120, 165-174 (1998), DOI:http://dx.doi.org/10.1115/1.2826955

Tang, L., et al., "Optimal Cooling System Design for Multi-cavity Injection Molding", Finite Elem. Anal. Des., 26, 229-251 (1997), DOI:http://dx.doi.org/10.1016/S0168-874X(96)00083-2

Viana, J. C.: Mechanical Characterisation Of Injection Moulded Plates, $\mathrm{Ph}$. D. Thesis, University of Minho, Guimarães (1999)

\section{Acknowledgements}

This work was supported by the Portuguese Fundação para a Ciência e Tecnologia under grant SFRH/BD/28479/2006.

Date received: April 13, 2011

Date accepted: September 5, 2011

Bibliography

DOI 10.3139/217.2511

Intern. Polymer Processing

XXVII (2012) 2; page 1-11

(C) Carl Hanser Verlag $\mathrm{GmbH} \&$ Co. KG

ISSN 0930-777X

You will find the article and additional material by entering the document number IPP2511 on our website at www.polymer-process.com 